\title{
Preserving Peer Replicas By Rate-Limited Sampled Voting
}

\author{
Petros Maniatis, Mema Roussopoulos, TJ Giuli, David S. H. Rosenthal, Mary Baker, Yanto Muliadi \\ Computer Science Department, Gates Building 4A \\ Stanford University, Stanford, CA 94305-9040 \\ \{maniatis,mgbaker\}@cs.stanford.edu
}

\begin{abstract}
The LOCKSS project has developed and deployed in a world-wide test a peer-to-peer system for preserving access to journals and other archival information published on the Web. It consists of a large number of independent, low-cost, persistent web caches that cooperate to detect and repair damage to their content by voting in "opinion polls." Based on this experience, we present a design for and simulations of a novel protocol for voting in systems of this kind. It incorporates rate limitation and intrusion detection in new ways that ensure even an adversary capable of unlimited effort over decades has only a small probability of causing irrecoverable damage before being detected.
\end{abstract}

\section{INTRODUCTION}

Academic publishing is migrating to the Web [27], forcing the libraries that pay for journals to transition from purchasing copies of the material to renting access to the publisher's copy [23]. Unfortunately, rental provides no guarantee of longterm access. Librarians consider it one of their responsibilities to provide future readers access to important materials. With millennia of experience of physical documents they have techniques for doing so: acquire lots of copies of the document, distribute them around the world, and lend or copy them when necessary to provide access.

In the LOCKSS ${ }^{1}$ project (Lots Of Copies Keep Stuff Safe), we model the physical document system and apply it to the Web, providing tools for libraries to take custody of the material to which they subscribe, and to cooperate with other libraries to preserve it and provide access. The LOCKSS approach deploys a large number of independent, low-cost, persistent web caches that cooperate to detect and repair damage by voting in "opinion polls" on their content. The initial version of the system is described in [30]; it has been under test since 1999 at about 50 libraries world-wide. The system is expected to be in production use at a much larger number of libraries in 2004. The protocol used by the initial version will not scale, and analysis of the first design for a revised protocol [26] showed it to be insufficiently resistant to attack.

Distributed digital preservation, with its time horizon of many decades and lack of central control, presents both unusual requirements, such as the need to avoid long-term secrets like encryption keys, and unusual opportunities, including the opportunity to make some system operations inherently very time-consuming without sacrificing usability. We present a design for and simulations of a new peer-to-peer opinion polling

\footnotetext{
${ }^{1}$ LOCKSS is a trademark of Stanford University.
}

protocol, based on these characteristics and on experience with the deployed LOCKSS system. We plan to migrate it to the deployed system this year.

Digital preservation systems must resist both random failures and deliberate attack for long periods. Their ultimate success can be evaluated only in the distant future. Techniques for evaluating their design must necessarily be approximate and probabilistic; they share this problem with encryption systems. We attempt to evaluate our design in the same way that encryption systems are evaluated, by estimating the computational effort an adversary would need to achieve a given probability of the desired result. In an encryption system, the desired result is to recover the plaintext. In our case, it is to have the system deliver a corrupt copy of a document. These estimates can be converted to monetary costs using technology cost curves and the result can be compared to the value of the plaintext or document, respectively.

In this paper we introduce our design principles and the deployed test system, then describe an abstract model of the application on which our analysis and simulations are based. We present our new protocol and the attacks its adversary can mount, then describe the simulations and their results.

These simulations show a system that resists for decades an adversary capable of unlimited sustained effort, by preventing him from applying more effort to the system than his victims. The system has a high probability of detecting an attack that can cause permanent damage before that damage becomes irreversible, while producing very few false positives. We do not use long-term secrets or identities. We believe this new protocol and its underlying principles are novel and will prove useful in the design of other long-term large-scale applications operating in hostile environments.

\section{DESIGN PRINCIPLES}

The LOCKSS system, in common with other digital preservation systems, exhibits some unusual features. First, the system need not operate quickly. Its purpose is to prevent rather than expedite change to data. Second, the system must function properly for decades, without central control and despite possible interference from attackers. These features, combined with our experience building and maintaining other large-scale distributed systems, led to the design principles we use here: accept that storage is unreliable; secrets cannot be expected to remain so for long; systems should have inertia so they change 
no faster than necessary, and thus will fail as slowly as possible; third-party reputation information should be avoided; and intrusion detection should be inherent.

The LOCKSS design is very conservative, appropriately so for a preservation system. It applies these principles to achieve a high probability that even a powerful adversary will fail to cause irrecoverable damage without detection.

Storage is unreliable. We assume that in our timescale no storage is reliable [10]. We replicate all storage across peers. A peer either audits the replicas regularly and repairs damage when found, or refreshes the replicas regularly, and limits the effect of any damage. Note that write-once media are at least as unreliable as disks, eliminating alternate designs dependent on storing documents or their hashes on CD-R (in our current deployment the CD-R containing the peer software is the cause of the vast majority of errors).

No long-term secrets. Or, to quote Diffie [14], "The secret to strong security: less reliance on secrets." Long-term secrets, such as private keys, are too vulnerable for our application. These secrets require storage that is effectively impossible to replicate, audit, repair or regenerate. They are also likely to leak over time, and recovering from such leakage is extraordinarily difficult [12], [34]. Our peers need keep secrets only for the duration of a single poll. Without long-term secrets attackers may spoof the identity of peers, but by requiring evidence of recent effort we reduce the time during which stability of identity matters to a few poll durations (Section 5.3), and we use short-term secrets to reduce spoofing during a poll (Section 5). Polls last a few hours.

Use inertia. The goal of a digital preservation system is to prevent change. Some change is inevitable, and the system must repair it, but there is never a need for rapid change. A system that fails abruptly, without providing its operators with a warning in time for them to take corrective action and prevent total failure [32], is not suitable for long-term preservation.

By making all operations in the system inherently expensive, we provide the system with an analog of inertia, limiting the rate of change in the system according to the effort applied to it. Because even the operations involved in failure are inherently time-consuming, this makes it very hard for attackers to overwhelm the system quickly, and provides time for humans to prevent the attack from resulting in catastrophic failure. Rate limiting techniques have proved useful in other areas [36]; we have the opportunity to exploit them since we have no need for speed.

Avoid third-party reputation. Gintis et al. [21] show that cooperation evolves and is dynamically stable under plausible conditions in multi-player games. The requirement is that cooperation provide a benefit to the group as a whole greater than the net cost to the cooperating player, and that the cost of participating is higher for the uncooperative player than the cooperative player. Biologists call similar interactions "costly signaling"; the recipient can assess the honesty of a signal by observing the effort needed to generate it.

By analogy, our peers use direct evidence of recent effort [18] instead of third-party reputation information because by doing so we avoid complex trust arrangements, the need for long-term peer identity, and vulnerability to subversion of previously loyal peers.

We do not accumulate evidence of good behavior over time, which prevents an attacker "cashing in" a history of good behavior in low-value interactions by defecting in a single highvalue interaction [37]. Instead, we adjust the costs imposed on peers in each interaction to ensure that attackers must expend efforts comparable to the defenders' to make progress toward their goal.

To the extent to which a peer does maintain a history of another peer's behavior, that history is very simple, derived from direct observation, and acts only as a hint. The system survives the loss or corruption of this memory at peers.

Intrusion detection is inherent. Random damage to individual replica documents stored by peers is incoherent, resulting in polls that are either landslide agreement or landslide disagreement. An attacker attempting to change a preserved document requires coherent damage to replica documents across peers, which will result in polls that are more closely contested. Contested polls, and thus coherent damage, raise an inconclusive poll alarm and lead to detection of the attack.

Opinion polls are thus subject to a super-majority requirement: the attacker cannot win with a simple majority of votes but must achieve a super-majority. An individual poll can only change a single replica of a document; to achieve his goal the attacker must win a large number of polls in close succession each by a super-majority. If even one of these polls fails to reach the super-majority, the resulting alarm reveals the attacker's activities.

Even if the attackers succeed in gradually damaging more replicas, by doing so they dramatically increase their risk of detection as peers without damage enter into polls with the damaged peers unbeknownst to the attackers. This mechanism has a low rate of false positives (Section 8.3).

We believe this mechanism to be fundamentally more robust than layering an intrusion detection system on top of an application; it does however share with conventional intrusion detection systems the notion that repelling attacks on the system must be a cooperative effort between the software and the humans responsible for it.

\section{THE LOCKSS SYSTEM}

The LOCKSS system models librarians' techniques for physical documents to preserve access to e-journals, by making it appear to a library's readers that pages remain available at their original URLs whether or not they are available there from the publisher. We thus preserve access to the material via normal techniques such as links, bookmarks, and search engines. To do this, participating libraries run persistent web caches that:

- collect by crawling the journal web-sites to pre-load themselves with newly published material,

- distribute by acting as a proxy cache for the library's local readers, supplying the publisher's copy if it is available and the local copy otherwise,

- preserve by cooperating with other caches that hold the same material to detect and repair damage.

Caches cooperate by participating in "opinion polls" in a peer-to-peer network. In each, a sample of peers votes on the 
hash of a specified part of the content. Polls provide peers with two kinds of confidence:

- Authenticity; journal publishers do not currently sign the material they distribute, nor do they provide a manifest describing the set of files forming a given paper, issue or volume. Moreover, the crawling process is unreliable. Evidence that many peers independently obtained the same material is the best available guarantee that content is authentic.

- Integrity; no completely reliable long-term storage medium is available. Evidence that many peers have recently agreed with each other as to the material is the best available guarantee that content has been preserved.

If damage is detected, a peer cooperates with the damaged peer if it remembers the damaged peer agreeing with it about the material in question. It allows the damaged peer to recover a good copy by acting as a proxy in the same way as it does for local readers, providing either the local or the publisher's copy. This mechanism defends against two important problems endemic to peer-to-peer systems (Section 10.4.2):

- freeloading; the only benefit a peer obtains from the system is a repair and to obtain it the peer must have participated in the past,

- theft; a peer only supplies material to a peer that proved in the past that it had that material, so the system does not increase the risk of theft.

In this way LOCKSS peers provide a distributed, highly replicated, self-healing store of data. The system does not materially increase the risks that publishers already run. This is important; under the DMCA [19] publishers must give permission for libraries to preserve their material.

Library budgets are perennially inadequate [3]. To be effective, any digital preservation system must be affordable in the long term.

Minimizing the cost of participating in the LOCKSS system is essential to its success. Individual peers are built from lowcost, unreliable technology and require little administration, relying on cooperation with other caches to detect and repair failures. There is no need for off-line backups on removable media. Creating these backups, and using them when readers request access to data, would involve excessive staff costs and latencies beyond a reader's attention span [33].

\section{System MOdel}

To simplify the analysis of the new opinion poll mechanism we abstract out the relevant features of the LOCKSS system. We consider a population of peers each attempting to preserve one copy of one large document it obtained from a publisher. Eventually, the publisher stops making the document available; the document's survival becomes dependent on mutual cooperation among the peers. At intervals a peer determines whether its copy still agrees with the consensus by calling an opinion poll that involves a sample of the peer population. If the copy disagrees a repair is obtained from another peer. Each peer uses one of a number of independent implementations.

A poll consists of three phases. The poll initiator peer constructs a sample of the peer population and invites them into the poll. The invited peers construct votes by hashing the document then send them to the initiator, who compares them to his copy. If the vote tally shows his copy to be damaged, he repairs it.

\subsection{Definitions}

We make the distinction between the following types of peers in the rest of this paper:

- A loyal peer is one that follows the LOCKSS protocol.

- A damaged peer is a loyal peer with a damaged document copy.

- A healthy peer is a loyal peer with the correct document copy.

- A malign peer is part of a conspiracy of peers attempting to subvert the system.

In addition, a node is any host that enters into our descriptions, while a peer is any node that participates, whether maliciously or benignly, in the LOCKSS protocol.

\subsection{Communication Model}

LOCKSS peers communicate over the Internet. We make the following assumptions about the security, connectivity and capabilities of this network:

- A node can send an IP packet to any other node with a high probability of success.

- IP security is not widely deployed.

- Routers between the peer's network boundaries are incorruptible.

- Spoofing the source address of IP packets has negligible cost.

\subsection{Adversary Model}

Our assumed adversary, a conspiracy of malign peers, takes three possible forms:

- The nuisance form intends to waste time and resources at the loyal peers by raising alarms.

- The stealth form intends to avoid detection while changing the consensus on the contents of the document, by causing polls to agree by an overwhelmingly large margin on a faulty version of the document.

- The attrition form intends to prevent loyal peers verifying their replica for long enough for random damage to corrupt it. We only describe the single-poll aspects of such attacks; longer-term aspects require additional peer state and are covered in Section 10.4.1

Conservatively, we assume that the adversary is very powerful:

- Conspiracy. The malign peers all know each other. Any information known to one malign peer is known to all. The malign peers have a magically incorruptible good copy of the document and can behave in all respects as loyal peers.

- Masquerading. A loyal peer cannot detect that another peer executing the LOCKSS protocol is malign.

- Adversary is not resource-constrained. The adversary can increase the power of the conspiracy at any time by adding 
peers he owns anywhere in the network, and by compromising nodes located anywhere in the network, causing them to behave as malign peers, as computational resources, or as spoofers or eavesdroppers on their local networks.

- Eavesdropping. The adversary is aware of the existence and contents of packets originating or terminating on a network on which it controls a node, but not on other networks.

- Common vulnerabilities among peers. If an exploitable vulnerability is discovered in the implementation used by a subset of the peers the adversary can quickly take over all peers using that implementation, change their copies without calling any polls, or change their state.

Our analyses and simulations do not depend on identifying the cause of an individual peer becoming malign. We measure the adversary by the extent to which he can subvert loyal peers by any means.

\section{The Opinion Polling Protocol}

At intervals much shorter than the anticipated rate of undetected random damage, each peer acts as a poll initiator and invites some of its peers to participate in a poll on the contents of the document. Each participating peer produces a vote, containing a digest of that peer's copy of the document. The poll initiator tabulates all the votes it receives. If it agrees with the overwhelming majority of votes it is satisfied. If it disagrees with the overwhelming majority of votes, it attempts to repair its copy and then re-evaluates the votes, hoping now to agree with the overwhelming majority. If the initiator cannot establish overwhelming agreement on its, possibly repaired, copy it raises an alarm.

The poll initiator distinguishes between inner circle and outer circle participants. The initiator picks its inner circle from those peers it already knows. It picks its outer circle from peers it does not already know that are nominated by its inner circle. All participants vote in the poll. The initiator uses inner circle votes to determine the consensus of the poll, and outer circle votes to expand its list of known peers.

The initiator of a poll and each peer in its inner and outer circles communicate via unicast messages. Figure 1 shows a typical message exchange between a poll initiator and its participants. The message types are explained in following sections.

The first two messages include a Diffie-Hellman key exchange [15] so that the initiator and each participant share a different session key for a symmetric cipher. The content of all messages except the first between an initiator and a participant is thus inaccessible to an eavesdropper. We anticipate, but do not depend on, peers generating new key pairs for each poll.

The overall goal of our design is that there be a high probability of loyal peers being in the healthy state despite failures and attacks, and a low probability that even a powerful adversary could damage a significant proportion of the loyal peers without detection.

\subsection{Economic Model}

Our design goal imposes the following economic requirements:

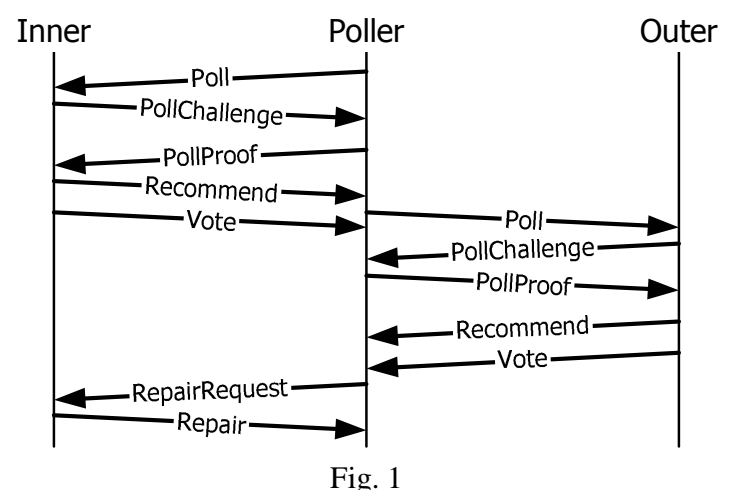

THE DIFFERENT TYPES OF PROTOCOL MESSAGES EXCHANGED DURING A POLL AMONG THE POLL INITIATOR, INNER CIRCLE PEERS AND OUTER CIRCLE PEERS. TIME FLOWS FROM TOP TO BOTTOM.

- It is expensive to vote. The cost of constructing a vote must be greater than the cost of verifying the vote. This prevents the construction of spurious votes, solely to waste the vote verifier's resources.

- It is expensive to be discovered. The price that a peer has to pay to be discovered by another peer must be greater than the cost of the damage that the former peer can inflict on the latter. This makes it expensive for the adversary to be in a position to attack, i.e., to persuade the poll initiator that the consensus is for his version of the document.

- It is expensive to initiate polls. The cost of a poll must be greater to the poll initiator than it is to all of the poll participants. This prevents polls being initiated with the sole purpose of wasting loyal peers' resources.

To fulfill these cost requirements, we interleave useful protocol operations with useless but provably performed computational effort. We employ a class of memory-bound functions [1], originally geared towards fighting spam, due to Dwork et al. [17]. These functions have a computation phase, yielding a proof of effort, and a verification phase, which checks its validity. A parameter of the system sets the factor by which the computation phase is more time consuming than the adjustable cost of the verification phase.

If an inconclusive poll alarm is raised human intervention is needed to determine which of the competing copies is the correct one and to restore it at peers that have been damaged. If the system is to be economically feasible, it must be hard for the nuisance adversary to cause an alarm (Section 5.6.4).

\subsection{Notation}

In the remainder of this section, and in subsequent sections, we use the following notation:

$N \quad$ Number of inner-circle peers invited into a poll

$V \quad$ Number of inner-circle peers whose vote is received

$Q \quad$ Quorum

$D \quad$ Maximum dissent allowable in a landslide poll

I Number of outer circle invitees per inner circle invitee

$M \quad$ Number of malign peers in $V$

$L \quad$ Number of loyal peers in $V$

A Maximum number of discredited votes without alarm 
$B \quad$ Average proportion of malign peers in loyal peer reference list

$S$

Number of CPU cache lines in the document

\subsection{Per-Peer State}

Each peer records other known peers in its reference list, a collection of entries of the form [peer IP address, time inserted]. Each peer is provided with a default list of peers, assumed to be made up from colleagues of the host librarian. This is used to initialize the reference list. The peer discards entries that survive several polls without taking part.

A spot in the reference list of a loyal peer has value. Inclusion of peer $X$ in peer $Y$ 's reference list indicates that peer $X$ recently participated in a poll called by $Y$, expending nontrivial resources doing so. We explain how a peer gets into a loyal peer's reference list in Section 5.6.3

\subsection{Poll Initiation Phase}

To start a new opinion poll, an initiator chooses a new, random poll identifier and $N$ peers from its reference list. These peers make up the inner circle of the new poll. The initiator sends a Poll message to each of them. It contains a newlygenerated public key for the initiator, and the poll identifier.

Inner circle peers respond with a PollChallenge message to the initiator. The message contains a newly-generated public key for the peer and a freshly-chosen random number, the challenge which is encrypted in the Diffie-Hellman session key. Inner circle peers who decline the invitation return a special challenge. All subsequent messages from the poll initiator to this participant are cryptographically dependent on the challenge and are thus unlikely to be replays.

The initiator waits for challenges either until it has received all of them, or until a timer expires. Then, for each inner circle peer who sent a challenge, the initiator computes a poll effort proof, returning it and the poll identifier to the peer in a PollProof message. The message proves that the initiator performed a predetermined amount of computational effort, cryptographically dependent on the poll identifier and challenge value. Its size, $C^{p}$, is set to outweigh the sum of all effort expended by poll participants in the remainder of this poll. The reason for this choice is discussed in Section 5.1 and suitable values for $C^{p}$ in Appendix $₫$

An inner circle peer can verify the poll effort proof cheaply, using its challenge and the poll identifier. If the verification succeeds, the peer nominates $I$ other peers at random from its own reference list, and returns them to the poll initiator in a Recommend message. The peer then proceeds to compute and send its vote, as described in Section 5.5

The poll initiator uses the recommendations it receives from its inner circle to compose its outer circle. It removes from each recommendation those peers that it already knows, then picks at random an equal number of peers from the recommendation of each inner circle peer. The initiator invites its outer circle peers exactly as it invited the inner circle, via an exchange of Poll, PollChallenge and PollProof messages. Any Recommend messages returned by outer circle participants are ignored.
The purpose of the outer circle is to expand the poll initiator's reference list with peers that have proven to him their investment in the system by constructing a valid vote. The larger the initiator's reference list, the smaller outer circle it will compose.

Duplicate identical challenges from the same IP address are ignored. Different challenges from the same IP address result in the exclusion of that IP address from the poll. An alarm is raised if there are several such conflicting challenges in a poll (Section 6.4).

\subsection{Voting Phase}

If they successfully verify the poll effort proof, inner and outer circle peers begin voting. This involves computing a cryptographic hash of the document and enough additional provable computational effort to meet our economic requirements (Appendix A.

Vote computation is divided into rounds which return a proof of computational effort and a hash of a portion of the document. The first round takes as input, and is dependent upon, the challenge and poll identifier. Subsequent rounds take as input, and are dependent upon, the output of the previous round. In each round, the size of the computational effort and the size of the document portion double. We make voters perform useless work (provable computational effort) before useful work (hashing of the document) to ensure that even a bogus vote costs at least as much to create as it does to verify.

The voter sends the proofs of computational effort and document hashes from each round in a single Vote message to the poll initiator. Identical duplicate votes are ignored. Differing but correctly encrypted votes from the same voter are discarded and cause the voter to be removed from the reference list.

The initiator collects votes until it has received all solicited votes, or until a timer expires. The vote collection timer is set based on the expected effort needed to compute a vote; if it is too short weaker peers cannot participate; if it is too long the adversary can "time-shift" its resources, computing many correct-looking votes.

\subsection{Vote Tabulation}

After collecting the votes, or after a repair, the poll initiator verifies the votes (Section 5.6.1) and tabulates the results. Verification deems a vote invalid, valid and agreeing with the initiator, or valid and disagreeing with the initiator. Invalid votes are discarded and their senders removed from the reference list.

If the number of valid votes $V$ is greater than the quorum size $Q$, the initiator counts the agreeing and disagreeing inner circle votes and acts as follows:

- Agreeing votes are no more than D. The initiator considers its current document copy damaged and repairs it (Section 5.6.2).

- Agreeing votes are at least $V-D$. The initiator considers its current copy the prevailing one (Section 5.6.3).

- Agreeing votes are more than $D$ but fewer than $V-D$. The initiator considers the poll inconclusive and raises an alarm (Section 5.6.4).

The protocol is conservative, in that a poll initiator makes decisions about its copy of the document only in response to 
the consensus of peers it chose itself. Peers recommended by others have to prove their investment in the system before they can affect a peer's actions.

If the initiator has not reached quorum $(V<Q)$ it cannot decide whether its copy is the prevailing one and it must schedule a new poll shortly. It can still expand its reference list by adding outer circle peers whose votes are valid and agreeing. A series of polls all of which fail to reach quorum will cause an inter-poll interval alarm.

5.6.1 Vote Verification: The initiator performs a number of vote verification rounds to check whether a vote is valid and, if so, whether it agrees with the poll initiator. The first round takes as input the challenge and poll identifier; succeeding rounds take the output of their predecessor. In each round, the computational effort included in the Vote message is verified based on the round input. If the effort proof is not correct, the vote is declared invalid. Otherwise, if the vote has yet to be declared disagreeing, the effort proof result and the document portion for the round are hashed. If the result does not match the hash in the vote, the vote is declared disagreeing and verification continues.

If all effort proofs are correct the vote is declared valid. If all document hashes match, it is declared agreeing.

5.6.2 Repair: If the initiator decides its copy is not the prevailing one, it attempts a repair by choosing an inner circle disagreeing voter at random, and sending it an encrypted RepairRequest message. If it receives a Repair message and obtains a new copy of the document it restarts the vote tabulation phase (Section 5.6, re-verifying the $V$ votes against the new copy and deciding how to proceed. If a repair disagrees with the vote of its provider then the vote, the voter and the repair are discarded.

The initiator need only make up to $D$ repair attempts; if in the process of any of the repair attempts, the initiator agrees with more than $D$ but fewer than $V-D$ voters in total it knows that it cannot possibly reach a conclusive win through subsequent repairs and can consider the poll inconclusive.

Repairs are the only interaction among peers involving the transfer of significant amounts of data and are implemented via an encrypted TCP stream.

5.6.3 Agreement: When the initiator concludes that its current copy of the document is the prevailing one, whether during the first tabulation or after a repair, it removes all disagreeing voters and enough randomly chosen agreeing inner circle voters to total $Q$. It then adds to the reference list the outer circle agreeing voters recommended by inner circle peers who actually voted, and a few peers randomly chosen from its default list. It then resets the time of insertion for the remaining agreeing inner circle voters.

By "forgetting" those peers on whose opinions a decision was made, the initiator ensures that it will consult different inner circle peers in its next poll. By adding a few of the default peers in addition to the outer circle agreeing voters the initiator dilutes the effect of the recommendations on the reference list. Without an adversary, the statistical properties of the default list and the outer circle should be similar. During an attack, their properties will be very different. The small dilution prevents even a very powerful adversary from completely controlling the reference list.
The adaptive behavior of the outer circle selection part of the poll initiation algorithm (Section 5.4) acts to reduce the length of long reference lists and increase the length of short reference lists. This reduces the long-term impact of differences in initial reference list configurations (Section 8.2.

5.6.4 Alarms: A peer raises an alarm when it determines that a poll is inconclusive. or suspects local spoofing, or has been unable to complete a poll for a long time. The alarm requests human involvement in suppressing an attack on the system. Raising an alarm is expensive; a significant rate of false alarms would render the system useless (Section 8.3.

An inconclusive poll alarm suggests that the library should contact others, examine the differences between their copies and determine a cause. Any compromised nodes found during this process should be repaired. If the institutions hosting the peers voting for bad copies cannot be identified or do not cooperate, their peers should be blacklisted.

A local spoofing alarm suggests that the network surrounding the peer should be audited and compromised nodes, if any (Section 6.5), removed. The cost of this alarm can be reduced by placing peers on their own subnets.

An inter-poll interval alarm is raised if no poll has reached quorum in several average inter-poll intervals. It suggests that an attrition attack is underway. Logs of poll requesters should reveal large numbers of requests from previously unknown peers, who should be blacklisted.

The expectation is that alarms will result in enough degradation of the adversary's infrastructure in terms of malign peers and compromised nodes that a rational adversary will be highly motivated to avoid them.

\subsection{Economics of Polls}

In Appendix $\mathrm{A}$ we derive the following computational effort sizes for poll initiation and for voting relative to $S$, the size of the document in cache lines. Conservatively, we estimate the cost of hashing as the cost of the $S$ cache misses required, assuming the document is in memory for the poll.

- Cost of hashing the document, $H \approx S$

- Cost of constructing a poll invitation per invitee, $C^{p}=$ $(20 / 3) S$

- Cost of verifying each poll invitation, $V^{p}=(5 / 3) S$

- Cost of computing a valid vote, $C^{v}=5 S$

- Cost of verifying an agreeing vote, $V^{v a}=2 S$

- Cost of verifying a disagreeing vote, $V^{v d}=S$

As an example, if the cost of hashing the document is 120 seconds, then the cost of verifying an agreeing vote is $240 \mathrm{sec}-$ onds, the cost of producing a vote is 600 seconds, the cost of verifying a poll invitation is 200 seconds and the cost of calling a poll is 800 seconds per invitee, or 4.5 hours for 20 invitees. An entire poll without repairs would thus last about 6 hours, comparable with the duration of polls in the currently deployed LOCKSS system.

The otherwise useless effort in poll initiation limits the rate at which a peer can call polls. This is important because the adversary can damage a loyal peer only when that peer calls a poll. The rate at which an attack can make progress is limited by the smaller of the adversary's efforts and the efforts of his 
victims. It is this property that enables the system to resist an adversary who is not resource-constrained (Section 77.

We choose to share the cost of a poll about equally between the initiator and the voters as a whole. If there are no malign peers in loyal peer's reference lists, the entire cost of polls is borne by the defenders. Malign peers will invite only loyal peers into the polls they call, so the cost will be borne equally by the adversary and the defenders. If the loyal peer's reference lists contain only malign peers, the cost of the polls they call will thus be borne equally by the adversary and the defenders. At intermediate levels of degradation, the cost will be borne more by the defenders than the adversary. In the worst case, our choice means the adversary can apply no more effort to the system than the defenders do, a counter-intuitive but valuable result.

The adversary cannot avoid the cost of calling polls. Malign peers need loyal peers to ask them for repairs. There is no point in a loyal peer asking for a repair from a peer in whose polls it has never voted, since it has no reason to expect the peer to remember its participation and thus to supply the repair.

\section{ATTACKS}

We divide the description of the attacks according to the role played in the poll by the malign peer. For each role, we examine attacks that affect the result of the poll, and those that degrade the quality of the initiator's reference list. We also describe additional attacks that deny service, and some spoofing attacks.

For the description and simulation of the opinion poll mechanism we assume the only state that persists between polls is the peer's reference list. Detecting and resisting the additional attacks requires peer state in addition to the reference list and is discussed in less detail in Section 10.4

\subsection{Poll initiator}

The worst a malign poll initiator can do is to deny service.

6.1.1 Affect poll result: A malign poll initiator can affect the result of a poll he initiates, but to no effect on the rest of the system, since he is already malign.

6.1.2 Degrade reference list: A malign poll initiator can degrade his own reference list, but to no effect on the rest of the system, since he is already malign.

6.1.3 Deny service: The attrition adversary acts as a malign poll initiator and invites large numbers of loyal peers into polls to waste their resources and prevent them coming to consensus. We set the cost of poll initiation to make this attack very expensive (Section 5.7), and raise an inter-poll interval alarm if we detect it.

\subsection{Inner circle}

A malign peer invited into the inner circle of a loyal peer's poll can take part in all three kinds of attacks.

6.2.1 Affect poll result: If enough malign peers are invited into a poll they can affect the result without detection. We refer to a poll of this kind as vulnerable and it must meet three conditions:

$$
M>Q-L
$$

$$
\begin{aligned}
M & >L \\
L & \leq D
\end{aligned}
$$

In other words, there must be enough malign and loyal votes to satisfy the quorum, there must be more malign than loyal votes, and the loyal votes must be few enough to avoid triggering an alarm.

A stealth adversary without an eavesdropper local to the poll initiator knows $M$ precisely but only has estimates of $L, Q$, and $D$. Real attackers thus run some risk of detection each time they attack a poll.

The nuisance adversary attacks a poll if it meets the alarm criterion:

$$
M>D
$$

Loyal peers are resource-constrained and cannot participate in every poll to which they are invited. It is legitimate for a peer to decline an invitation. The stealth adversary will attack only when he has high confidence that a poll is vulnerable. His malign peers could remain in a loyal peer's reference list without further effort by declining all invitations until he decided to attack, were it not for the limit on the amount of time any peer can live in a peer's reference list without accepting an invitation and voting (Section 5.3.

6.2.2 Degrade reference list: A malign inner circle invitee has the opportunity to degrade the quality of the initiator's reference list, by recommending other malign peers into the poll's outer circle. Malign peers recommended for the outer circle will only get into the loyal poll initiator's reference list if they expend effort and vote with the consensus.

However, the malign peers have an advantage over the loyal peers. They know the identities of the other malign peers. The loyal peers will recommend $B$ malign peers on average, but the malign peers will recommend only malign peers. Doing so costs the adversary effort over time because the malign invitees have to exert effort to get in and stay in the loyal peer's reference list. The loyal peers also have to exert effort to stay in the malign peer's lists, even if this particular effort is wasted. The malign peers do not have to exert effort to stay in their coconspirator's lists.

6.2.3 Deny service: A malign inner circle peer can do four things to deny service, only the last of which is effective:

- It could generate a bogus vote in a poll the malign peers are certain to lose. The initiator will eliminate the vote with much less effort than it took to create and remove the malign peer from its reference list. This is not a good strategy.

- It could recommend loyal peers into the outer circle then discredit the poll by inviting the same loyal peers into a bogus poll with the same ID. The effort of creating the bogus poll is larger than the cost to the these loyal peers of detecting that it is bogus. The adversary would benefit more from using the effort to degrade the initiator's reference list further.

- It could refuse to send a vote, raising the probability that the poll would fail to get a quorum, at the risk of losing its slot in the initiator's reference list if the poll does achieve 
a quorum. Unless the adversary is resource-constrained the malign peer should vote and recommend other malign peers.

- If there are more than $A$ malign peers in the inner circle they can jointly mount a "phantom spoofer" attack (Section 6.5 by sending multiple challenges or votes, thus discrediting themselves. The effect is to raise an alarm at the initiator, wasting human time and money.

\subsection{Outer circle}

A malign peer invited into the outer circle of a poll is offered a chance to occupy a place in the loyal peer initiator's reference list. This is a valuable opportunity to attack a future vulnerable poll.

6.3.1 Affect poll result: A malign outer circle invitee cannot change the result of the poll because the initiator will not consider its vote.

6.3.2 Degrade reference list: To degrade the reference list, the malign outer circle invitee must vote with the consensus. It must then continue to act as a healthy peer in future polls until the malign peers decide to attack.

6.3.3 Deny service: If the malign outer circle invitee is one of more than $A$ total malign invitees, they could mount the phantom spoofer attack by sending duplicate different challenges or votes.

A malign outer circle invitee could also remain mute, causing a shortage of outer circle participants, but the adversary would benefit more from degrading the initiator's reference list.

\subsection{Spoofer and Eavesdropper}

A spoofer on the same subnet as a peer is strictly more powerful with respect to that peer than a spoofer on another peer's network. A spoofer local to either of the peers can prevent the initiator receiving the PollChallenge by flooding the sender, and substitute himself for the invitee.

6.4.1 Affect poll result: Malign peers without an eavesdropper local to the poll initiator cannot attack a poll without running some risk of detection (Section 6.2). The eavesdropper can reduce but not eliminate this risk by examining the source and destination addresses of Poll and PollChallenge messages and identifying the loyal invitees. Some will decline to participate, but encryption will prevent the eavesdropper knowing how many. Some will commit to vote but will fail to do so. The adversary thus has an upper bound for $L$ but still only estimates for $Q$ and $D$; some risk remains in every attack.

If challenges from loyal inner circle invitees are lost through UDP packet loss or packet flooding attacks, a spoofer local to the initiator can substitute for the invitees and possibly affect the result. Further, the spoofer hears the invitations, and thus knows which loyal peers to flood with packets in an attempt to cause their challenges to be lost. Similarly, if a challenge from a loyal inner circle invitee is lost, a spoofer local to the invitee can substitute for this vote. Both attacks can be made unlikely to evade detection by requiring loyal peers whether or not they decline an invitation to re-send their PollChallenge message at random intervals through the duration of the poll.
A spoofer or eavesdropper local to a peer is thus fairly powerful, but only relative to that peer. An adversary powerful enough to maintain compromised hosts local to a large proportion of the peers is powerful indeed.

6.4.2 Degrade reference list: A spoofer on the same subnet as a loyal peer poll initiator can increase the rate of degradation of the loyal peer's reference list by spoofing the source address of fewer than $A$ loyal invitees to each poll in bogus PollChallenge messages. The initiator will not know which is the real invitee's message and public key.

6.4.3 Deny service: A spoofer on the same subnet as a loyal peer initiator can deny service by discrediting challenges as above, causing polls to fail. Doing so requires discrediting more than $A$ challenges, triggering an alarm and wasting the investment in acquiring a spoofer on this network.

\subsection{Phantom spoofer attack}

Malign invitees can discredit their own challenges to polls initiated by loyal peers. If more than $A$ do so a false alarm is raised, suggesting that there is a spoofer local to the victim. The adversary causes wasted time and effort since human intervention at the victim is required.

Once a human has investigated and ruled out the possibility of a local spoofer, the peers that discredited their own votes are shown to be malign, or to have a local spoofer, and are blacklisted.

\section{DeFENSES}

Calling and participating in polls are both expensive, and a peer's resources are limited. There is thus a limit on the rate at which a loyal peer can call polls and on the number of simultaneous polls in which it will participate. These rates, the quorum, and the total population limit the total rate at which polls can be called by the loyal peers.

The poll calling rate, the rate of expiration of entries in reference lists, and the limited number of recommendations of outer circle peers by inner circle peers indirectly set a limit on the length of a loyal peer's reference list. We have evidence that although creating identities is cheap, the adversary gets little or no benefit from doing so beyond the number needed to saturate a loyal peer's reference list.

To turn a good copy bad, the malign peers need to win a poll called by a healthy peer. Our choice of poll cost parameters (Section 5.7 limits the adversary to applying no more effort to the system than the defenders. Irrespective of the effort available to them, there is thus a limit on the rate at which the malign peers can exert it to damage good copies, and thus a limit to the rate at which the system can succumb even to an adversary with no resource constraint.

A healthy peer that loses a poll and becomes damaged does not stop participating in polls. The stealth adversary must continue to exert effort to prevent damaged copies from being repaired. The quality of the victim's reference list will determine the result of the next poll after an attack on a vulnerable poll turns the copy bad. There are three possible outcomes of such polls, the copy remains bad, it reverts to being good, or an alarm 
goes off, depending on the balance of healthy, damaged and malign peers invited into the poll: To be pessimistic we equip our simulated adversary with an oracle that reveals $L, Q$ and $D$.

- If there are at least $(Q-D)$ malign peers the poll is vulnerable, the oracle will reveal this to the malign peers and they will attack. The victim's copy will remain bad irrespective of the number of damaged peers.

- If there are at least $(Q-D)$ healthy peers the copy will become good because the healthy peers will win no matter what the malign peers do. The oracle will reveal this and the malign peers will not attack.

- Otherwise, there are fewer than $(Q-D)$ malign peers and despite the oracle they cannot be sure that an attack on the poll will be undetected. The oracle reveals $L$ but not the number of damaged peers. A stealth adversary will not attack the poll. There are three cases:

- If there are at least $(Q-D)$ damaged peers, the copy will remain bad.

- If there are fewer than $D$ damaged peers, the copy will become good.

- Otherwise the alarm will go off and the adversary will be detected.

The probability that a damaged peer will be repaired thus sets a lifetime for the damaged copy. The probability of reader encountering a damaged copy depends on the rates of both corruption and repair, which in turn depend on the efforts actually expended by the adversary and the loyal peers.

\section{Simulation AND Results}

We demonstrate the following points:

- If the system is not attacked, substantial rates of random damage result in low rates of false alarms (Section 8.3.

- A nuisance adversary whose goal is simply to raise an alarm has to exert significant effort over a long period (Section 8.4).

- A stealth adversary whose goal is to avoid detection while corrupting the document by winning polls will fail (Section 8.5.

- An attrition adversary whose goal is to prevent consensus long enough for random damage to corrupt the document will be detected before he succeeds (Section 8.6).

\subsection{Simulator}

We use a flow-based simulator [22] designed for scalability over large numbers of nodes, large amounts of traffic, and long periods of time. It supports a variety of network models allowing trade-offs between speed and accuracy. Our simulations span up to 30 (simulated) years, so we chose a faster network option, modeling propagation latency but not traffic congestion. The simulator can also model expensive computations, allowing realism in the cost of hashes and proofs of effort.

\subsection{Simulated Environment}

Our adversary is a distributed system with instantaneous self awareness; his strategy is effected instantly via any of his components. He can send and receive messages from any number of IP addresses in addition to his initial group of malign peers. We assume that he has sufficient real resources to perform any computation required by the protocol of these peers.

In the nuisance and stealth cases, we simulate the system from start to finish of an attack. The attack starts when a vulnerability common to a fraction $F$ of the peers allows the adversary to subvert them. The other $(1-F)$ of the peers remain loyal. These simulated malign peers call polls at the same rate as loyal peers (Section 5.7), and participate in as many polls as needed; the adversary assigns resources to malign peers to have the maximum effect on the system. In the attrition case, the adversary uses throw-away IP addresses to call polls as fast as his resources permit, and does not vote in any polls.

Our simulations use a population of 1000 peers each storing a document taking 120 s to hash. The other parameters are $N=20, Q=10, D=3, A=3$. Each peer's reference list is initialized using a clustering technique. Peers are randomly assigned to clusters of 30 peers, and links randomly created to provide each with 29 other peers, $80 \%$ of which will be in their cluster and the rest in other clusters.

We have verified that our results are not dependent on the initial reference list configuration by running simulations with three different initial configurations of loyal peer reference lists:

- A uniform sample of the peer population.

- The clustering technique above.

- A power-law distribution containing a small proportion of highly-connected peers, which mirrors the Internet topology [20].

Using traditional metrics of graph characterization, such as average shortest path, clustering coefficient, and degree distribution we examined the evolution of the peer network and found that the three initial configurations converge to similar graphs under these metrics.

\subsection{Rate offalse positives}

Our peers are typically implemented in low-cost technology, lacking features such as ECC memory. They are thus subject to random undetected errors. In over 200 machine-years of the test deployment, we have observed one peer in which these errors became noticeable.

Without an adversary, but with peers subject to random damage they do not themselves detect, Figure 2 shows that false alarms occur rarely. The simulations cover 20 years with $e v$ ery peer suffering undetected damage on average every 5 to 9 machine-years. Over 20 runs, we show the minimum, average and maximum total rates of false alarms (i.e. raised at any peer in the entire system). With undetected damage at each peer every 5 years, in the worst case some peer will see an alarm on average every 44 days, and the average peer will see an alarm once in about 120 years.

\subsection{Nuisance Adversary}

Figure 3 shows the effect of a nuisance adversary with 1 to 128 nodes-worth of computing effort available who subverts 1 to 64 peers then attempts to raise an alarm at any peer. The simulation ends after 3 years or at the first such alarm. The error bars show minimum, average and maximum times to the 


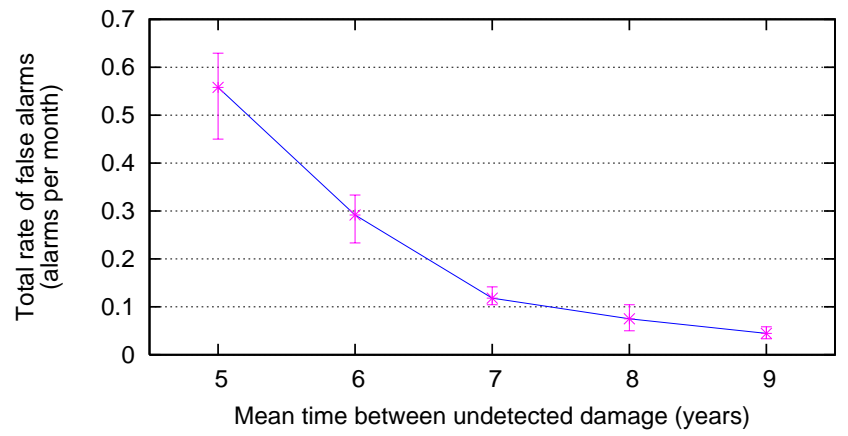

Fig. 2

THE TOTAL RATE OF FALSE ALARMS VERSUS THE MEAN TIME BETWEEN UNDETECTED DAMAGE AT EACH PEER, WITH NO ADVERSARY.

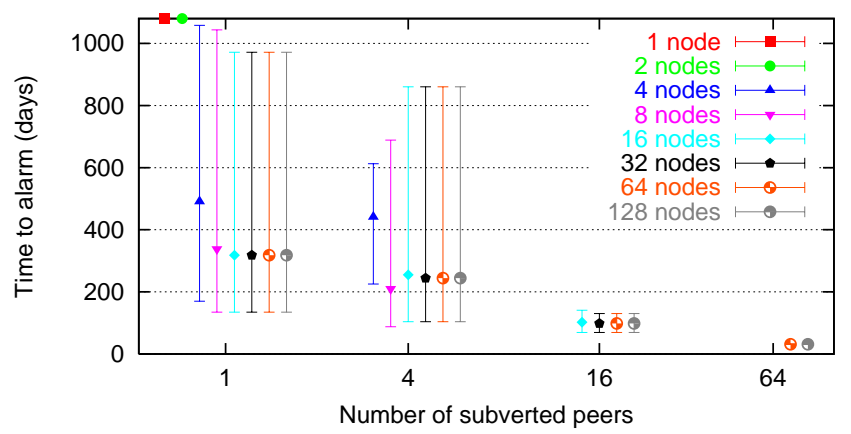

Fig. 3

TIME FROM START OF NUISANCE ATTACK TO FIRST ALARM AT ANY PEER AGAINST NUMBER OF PEERS SUBVERTED, FOR VARYING ADVERSARY RESOURCES. SOME ATTACKS WITH FEW SUBVERTED PEERS DO NOT CAUSE ALARMS IN OUR TIMESCALE.

first alarm, over 12 runs per data point, with some attacks not generating an alarm in the first 3 years.

If the nuisance adversary subverts only a few peers, irrespective of his computing resource, he takes about 6 months to raise an alarm, or (see Figure 2) the equivalent of a random damage rate of once every 6-7 years. If he takes over a large number of peers, irrespective of his computing resource the alarm happens quickly. This seems to be suitable behavior, in that large compromises should be detected.

\subsection{Stealth Adversary}

We show that a stealth adversary with infinite resources, whose goal is to avoid detection while corrupting the document, will fail. We simulate this attack in two phases. In the first phase the adversary subverts varying proportions of the peer population and then lurks for up to 20 years, degrading the reference lists of the loyal peers. At some badness (average proportion of malign peers in loyal peers' reference lists), the second phase starts. The adversary attacks vulnerable polls for 10 years or until the alarm goes off. We show four graphs:

- Figure 4 shows the minimum time taken by the lurking phase to achieve various badness levels. The $x$ axis shows the proportion of total peers that are initially subverted.

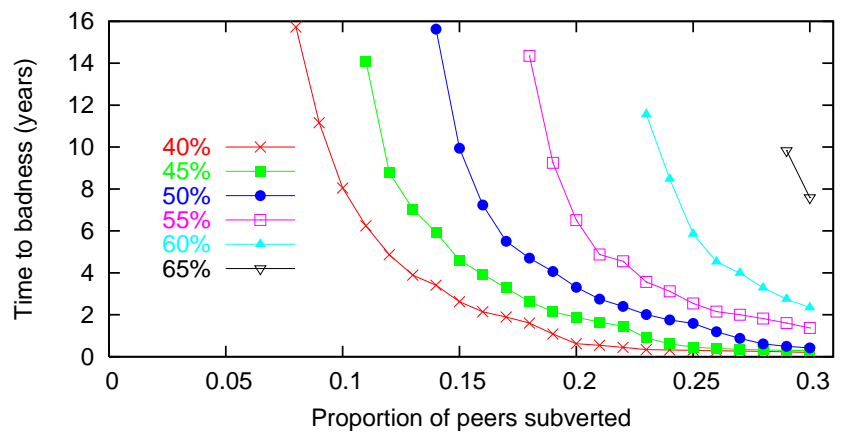

Fig. 4

Minimum TIME FOR THE STEALTH ADVERSARY TO ACHIEVE VARIOUS BADNESS LEVELS, STARTING WITH VARIOUS PROPORTIONS OF INITIALLY SUBVERTED PEERS IN THE SYSTEM.

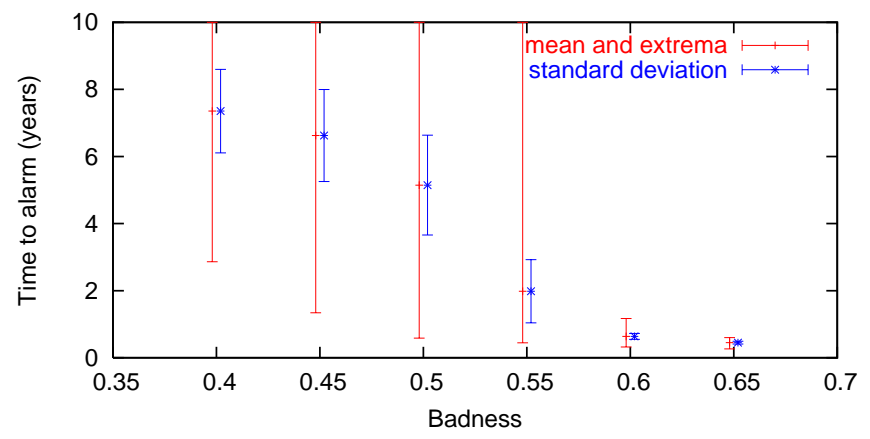

Fig. 5

TIME FROM START OF ATTACK PHASE FOR AN INFINITELY POWERFUL STEALTH ADVERSARY TO THE TIME OF DETECTION, FOR DIFFERENT REFERENCE LIST BADNESS LEVEL.

The $y$ axis shows the minimum time it takes the adversary to deteriorate the loyal peers' reference lists to various badness levels, over two runs per subversion level. We graph different curves for various badness levels. Each run lasts 20 simulated years. Note that runs with low subversion levels do not achieve any of the shown badness levels in 20 years.

- Figure 5 shows how long the attack phase lasts before it is detected. For each badness, we show the average, minimum and maximum times until detection, as well as the standard deviation. These statistics are calculated over 10 runs for each subversion level that achieved the indicated badness.

- Figure 6 shows the effect of the attack phase. We graph the maximum proportion of damaged peers across all runs, as a function of time from the start of the attack phase up to 10 years. The upper line shows the proportion of attacks that have not yet caused an alarm at a particular time. For example, at the end of the 10 years (3600 days) during which we simulate attack phases, about $20 \%$ of the runs had not raised an alarm, but had managed to damage only $5 \%$ of the document copies of loyal peers. The maximum damage achieved by any attack is about $20 \%$; attacks this successful do not last long. 


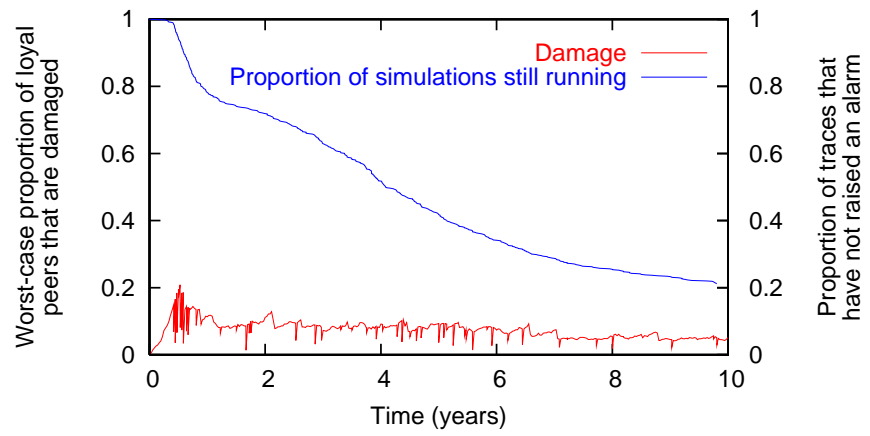

Fig. 6

WORST-CASE PROPORTION OF LOYAL COPIES THAT THE STEALTH ADVERSARY DAMAGES AS A FUNCTION OF TIME DURING THE ATTACK PHASE, OVER ALL RUNS.

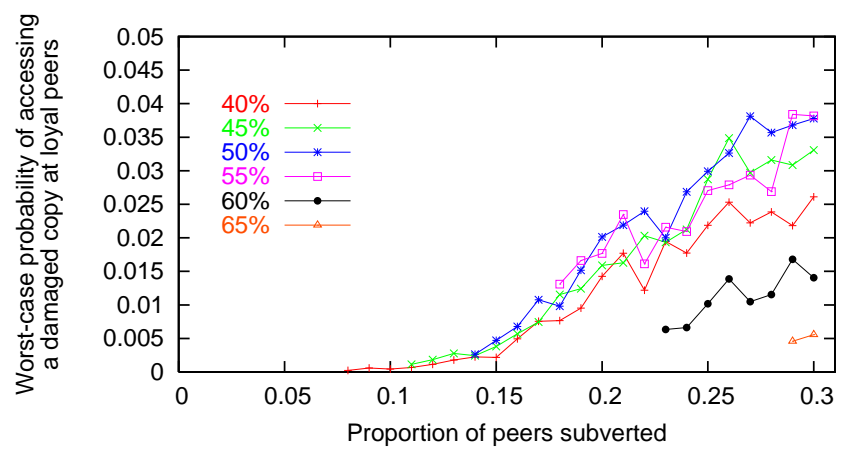

Fig. 7

ADDITIONAL PROBABILITY OF RANDOM READER SEEING A DAMAGED COPY AS A FUNCTION OF REFERENCE LIST BADNESS AT THE START OF

THE ATTACK PHASE.

- Figure 7 7 combines the previous two experiments to summarize the effect of the attack. On the $x$ axis, we show the proportion of peers in the system that are subverted by the adversary. On the $y$ axis, we show the worst-case additional probability that a reader who accesses a document will find a damaged copy, above that caused by the initial subversion. We graph a different curve for each badness at which the adversary switches from lurking to attacking.

We see that the longer the adversary lurks and thus the higher proportion of the loyal peers' reference lists that is malign, the greater his effect on the system. Nevertheless, with unlimited effort, unlimited identities, a vulnerability that allows him to subvert $30 \%$ of the peers, and an oracle, the worst the stealth adversary can do is to increase the $30 \%$ probability that a reader at a random peer will see a damaged copy he achieved by his initial $30 \%$ subversion, to $34 \%$ after about 2 years sustained effort.

\subsection{Attrition Adversary}

We simulate the attrition adversary with unlimited identities but limited resources. He calls useless polls to consume the loyal peers' resources. Unlike the stealth adversary, he neither lurks to degrade reference lists nor attacks polls. Loyal peers give priority to calling their own polls, their remaining

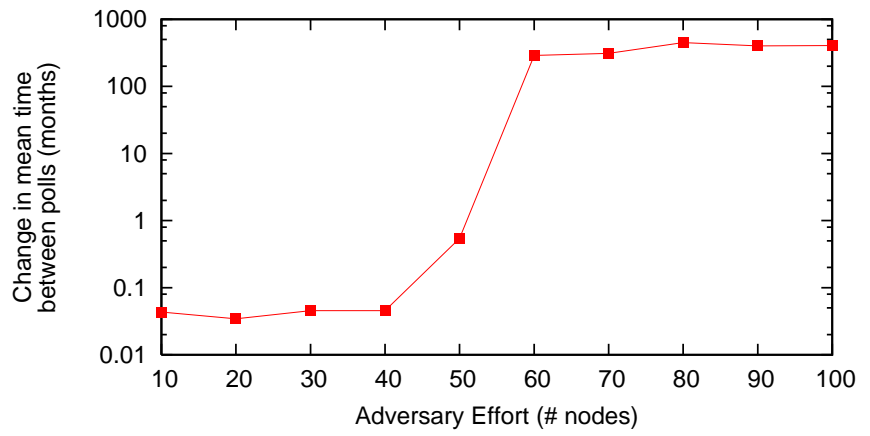

Fig. 8

EFFECT OF THE ATTRITION ADVERSARY IN INCREASING MEAN TIME BETWEEN POLLS AS A FUNCTION OF HIS EFFORT. THE $y$ AXIS IS IN LOGARITHMIC SCALE.

resources go to participating in polls called by others. A loyal peer will raise an alarm if it hasn't completed a poll in 3 times the expected inter-poll interval.

Figure 8 shows the increase in the average inter-poll time at loyal peers as a function of the effort expended by the attrition adversary. Without sufficient resources, he has little effect on the system. If he can deploy sufficient resources, in our case about 50 machines, he can prevent polls reaching quorum and will be detected after 3 average inter-poll intervals ( 9 months), which is much less than half any reasonable mean time between random undetected damage at a peer. Any damage accumulated will therefore be repaired after the response to the alarm suppresses the attack.

If the attrition adversary with few resources focuses his attention on a single peer he can cause it to raise an alarm after about 9 months. This might be an effective way to deny service; further work is needed to prevent it (Section 10.4.1).

\subsection{Phantom spoofer attack}

We are currently re-running the nuisance adversary simulations of Section 8.4 with a different vulnerability criterion, corresponding to the ability of the malign peers to cause a phantom spoofer alarm at a loyal peer (Section 6.5). The results will be available shortly.

\section{RELATED WORK}

In common with the Byzantine-fault-tolerance (BFT) literature (e.g., [5], [6], [8], [25], [29]), our voting protocol derives an apparent prevailing opinion among a set of peers, some of whom are malicious. There are many differences; our population size is too large for BFT's global communication, we degrade gradually rather than mask the effects of failure or attack, and because we cannot assume an absolute upper bound on the malicious peers' resources we have to consider the possibility of being overwhelmed. We use sampling to avoid global knowledge or communication, rate-limiters to prevent our adversary's unlimited resources overwhelming the system quickly, and integrated intrusion detection to preempt unrecoverable failure. 
Our work has similarities with the anti-entropy protocol forming part of Bimodal Multicast [4], a reliable multicast protocol in which peers send digests of their message histories to randomly chosen other peers. Peers receiving these messages can detect missing messages and request repairs from the peer that sent the digest. The system's name comes from its bimodal distribution of delivery probability, which is similar to our distribution of poll results absent an attack. As in our case, it exploits the properties of random graphs. As the authors acknowledge, the lack of voting among the peers appears to leave the anti-entropy protocol vulnerable to malign peers.

Our work also shares a goal with some of the first peer-topeer systems including Freenet [9], FreeHaven [16], and the Eternity Service [2], namely to make it hard for a powerful adversary to damage or destroy a document in the system. The other key goal of these systems is to provide anonymity for both publishers and readers of content, which we do not share. It would make our system both illegal and unworkable, since we preserve content that must be paid for.

Several studies have proposed a persistent, peer-to-peer storage service including Intermemory [7], Oceanstore [24], CFS [11], PAST [31], and Tangler [35]. Some (e.g. Oceanstore) implement access control by encrypting the data and thus do not solve our preservation problem, merely reducing it from preserving and controlling access to the content, to preserving and controlling access to the encryption key. Others (e.g. PAST) implement access control based on long-term secrets and smartcards or a key management infrastructure. Neither is appropriate for our application. Some (e.g. Intermemory) use cryptographic sharing to proliferate $n$ partial replicas, from any $m<n$ of which the file can be reconstituted. Others (e.g. PAST) replicate the entire file, as we do, but do not allow control over where the replicas are located. The goal of the LOCKSS system is to allow librarians to take custody of the content to which they subscribe. This requires that each library keep its own copy of a document it has purchased, not share the load of preserving a small number of copies.

Moore et al. [28] have some interesting measurements on packet-level denial-of-service attacks, types, and frequencies. For example, most attacks are relatively short with 90 percent lasting less than an hour. Rarely do such attacks span multiple days. The relevance of this data to application-level denialof-service attacks is questionable, but our simulated attacks require attention spans from the attackers several orders of magnitude longer.

\section{FUTURE WORK}

We have as yet simulated only the basic mechanism of opinion polling; we are a long way from a deployable implementation. Apart from many practical details, such as applying the mechanism to collections of documents, we see a number of areas of broader interest.

\subsection{Malign Peer Strategies}

An important question for the future is whether we can design a better adversary. The design of our simulated adversary is conservative, exploiting his advantages including an oracle a real attacker would lack, and worst-case reference list quality at the loyal peers. Nevertheless, we have not proven the strategies he uses to be optimal.

\subsection{Setting System Parameters}

We have examined only a single cost structure, sharing the cost of polls about equally between caller and voters, and using on average $1 \%$ or so of a peer's resources for one document. There are reasons to believe placing more of the cost on the caller and making the cost higher overall would be beneficial in suppressing the attrition adversary.

Furthermore, although we show that for a reasonably sized network, system parameters can be set appropriately, we have not explored the parameter space or identified optimal strategies for setting those parameters.

\subsection{Imaginary friends defense}

Section 6.4 shows that a loyal peer is somewhat vulnerable to a local eavesdropper or spoofer. A loyal poll initiator can turn the spoofer's weapons against them using the "imaginary friends" defense. It sends $N+X$ Poll messages, $N$ of which it actually wishes to pursue. The other $X$ messages are meant to confuse the eavesdropper. In response to the PollChallenge messages sent back by the $X$ nodes, the poll initiator replies with cheaply constructed, invalid PollProof messages. An eavesdropper will not know they are invalid because they are encrypted. The $X$ nodes easily detect they are invalid and take no further part in the poll. The poll initiator then spoofs the silent peer's source address in false encrypted Recommend and Vote messages. The eavesdropper believes a valid protocol interaction and loyal vote has occurred and raises his estimate of $L$. A real attacker without our oracle is thus less likely to decide the poll is vulnerable, less likely to attack, and less likely to cause or maintain damage.

There is a probability of $B$ that each of the $X$ peers is malign. An invalid PollProof sent to a malign peer will reveal that the initiator is using the "imaginary friends" defense, reducing its effectiveness by reducing the uncertainty it induces in the adversary. It is thus most effective when the adversary is relatively weak.

\subsection{Additional Peer State}

Restricting peer state only to the reference list leaves the system vulnerable to various kinds of long term misbehavior by malign or selfish peers. In particular, neither freeloading nor denial of service attacks can be detected except by statistical analysis of a series of polls, requiring additional peer state beyond the reference list itself.

10.4.1 Detecting Attrition: The 3-way poll initiation handshake means that the attrition adversary has to own the IP addresses he uses to request polls; spoofed addresses would not work. Our simulated attrition adversary with unlimited IP addresses is unrealistically powerful; a real attacker would have to use a limited number of addresses and could be detected by additional peer state. 
10.4.2 Freeloading and Theft: Section 5.7 sets a cost for a peer to call a poll, and to participate in a poll called by others. Why would peers incur either of these costs?

To prevent freeloading and theft, peers supply repairs only to those peers that agreed with them in past polls they initiated. Peers request repairs from other peers in whose past polls they participated. Despite the cost of doing so, a peer needs to call polls to detect damage to its copy and to maintain its list of peers who may be supplied with repairs. Despite the cost of doing so, a peer must also participate in polls called by others to spread and refresh knowledge of its legitimate possession of a copy so that it can later repair any damage to its copy. In neither case is the peer's memory critical to the system's operation although it is valuable to the individual peer.

This algorithm has been implemented in the deployed system but not even simulated with the new protocol.

10.4.3 Other Selfish Peer Strategies: A peer might vote correctly, taking little bandwidth, but refuse to pay the bandwidth costs of supplying a good repair copy on request. Additional peer state should provide ways of punishing this form of misbehavior.

\section{CONCLUSiON}

We have shown that a combination of massive replication, rate limitation, inherent intrusion detection and costly operations can produce a peer-to-peer system with remarkable ability to resist a range of different attacks by an extraordinarily powerful adversary over decades. Its lack of dependence on longterm secrets and stable identities blocks many of the paths by which real systems are attacked. We believe that this protocol will allow us to scale the deployed LOCKSS system to production levels in the next year. Despite the unusual characteristics of the application for which it was designed, in particular its long time horizon, we believe that the concepts and principles underlying this protocol will be useful in the design of other long-term large-scale applications operating in hostile environments.

\section{REFERENCES}

[1] Martín Abadi, Mike Burrows, Mark Manasse, and Ted Wobber. Moderately Hard, Memory-bound Functions. In Proceedings of the 10th Annual Network and Distributed System Security Symposium (NDSS), February 2003.

[2] Ross Anderson. The Eternity Service. http://www.cl.cam.ac.uk/users/ rja14/eternity/eternity.html.

[3] Assoc. Research Libraries. ARL Statistics 2000-01. http://www.arl.org/stats/arlstat/01pub/intro.html 2001.

[4] Kenneth Birman, Mark Hayden, Oznur Ozkasap, Zhen Xiao, Mihai Budiu, and Yaron Minsky. Bimodal Multicast. ACM Transactions on Computer Systems, 17(2):41-88, 1999.

[5] Miguel Castro and Barbara Liskov. Practical Byzantine Fault Tolerance. In Proceedings of the 3rd Symposium on Operating Systems Design and Implementation (OSDI 1999), New Orleans, LA, USA, February 1999. USENIX Association.

[6] Miguel Castro and Barbara Liskov. Proactive Recovery in a ByzantineFault-Tolerant System. In Proceedings of the 4th Symposium on Operating Systems Design and Implementation (OSDI 2000), pages 273-287, San Diego, CA, USA, October 2000. USENIX Association.

[7] Yuan Chen, Jan Edler, Andrew Goldberg, Allan Gottlieb, Sumeet Sobti, and Peter Yianilos. A Prototype Implementation of Archival Intermemory. In International Conference on Digital Libraries, pages 28-37, Berkeley, CA, USA, 1999.
[8] Benny Chor and Cynthia Dwork. Randomization in Byzantine Agreement. Advances in Computing Research, 5:443-497, 1989.

[9] Ian Clarke, Oskar Sandberg, Brandon Wiley, and Theodore W. Hong. Freenet: A Distributed Anonymous Information Storage and Retrieval System. In Hannes Federrath, editor, Proceedings of the Workshop on Design Issues in Anonymity and Unobservability, volume 2009 of Lecture Notes in Computer Science, pages 46-66, Berkeley, CA, USA, July 2000. Springer.

[10] ConservationOnline. Electronic Storage Media. http://palimpsest.stanford.edu/ bytopic/ electronic-records/ electronicstorage-media/.

[11] F. Dabek, M. F. Kaashoek, D. Karger, R. Morris, and I. Stoica. Widearea Cooperative Storage with CFS. In Proceedings of the Eighteenth Symposium on Operating Systems Principles, 2001.

[12] Don Davis. Compliance Defects in Public-Key Cryptography. In Proceedings of the 6th USENIX Security Symposium, pages 171-178, San Jose, CA, USA, July 1996.

[13] Drew Dean and Adam Stubblefield. Using Client Puzzles to Protect TLS. In Proceedings of the 10th USENIX Security Symposium, Washington, DC, USA, August 2001.

[14] Whitfield Diffie. Perspective: Decrypting The Secret to Strong Security. http://news.com.com/2010-1071-980462.html January 2003.

[15] Whitfield Diffie and Martin E. Hellman. New Directions in Cryptography. IEEE Transactions on Information Theory, IT-22(6):644-654, November 1976.

[16] Roger Dingledine, Michael J. Freedman, and David Molnar. The Free Haven Project: Distributed Anonymous Storage Service. In Proceedings of the Workshop on Design Issues in Anonymity and Unobservability, 2000.

[17] Cynthia Dwork, Andrew Goldberg, and Moni Naor. On Memory-Bound Functions for Fighting Spam. Manuscript, 2002.

[18] Cynthia Dwork and Moni Naor. Pricing via Processing. In Advances on Cryptology (CRYPTO 1992), volume 740 of Lecture Notes in Computer Science, pages 139-147. Springer, 1993.

[19] Electronic Frontier Foundation. Digital Millennium Copyright Act (DMCA) Archive. http://www.eff.org/IP/DMCA/

[20] Michalis Faloutsos, Petros Faloutsos, and Christos Faloutsos. On Powerlaw Relationships of the Internet Topology. In Proceedings of SIGCOMM 1999, 1999.

[21] Herbert Gintis, Eric Alden Smith, and Samuel Bowles. Costly Signalling and Cooperation. J. Theor. Biol., 213:103-119, 2001.

[22] TJ Giuli and Mary Baker. Narses: A Scalable, Flow-Based Network Simulator. Technical report, Computer Science Department, Stanford University, Stanford, CA, USA, November 2002.

[23] Michael Keller, Victoria Reich, and Andrew Herkovic. What is a Library Anymore, Anyway? Manuscript, 2003.

[24] John Kubiatowicz, David Bindel, Yan Chen, Steven Czerwinski, Patrick Eaton, Dennis Geels, Ramakrishan Gummadi, Sean Rhea, Hakim Weatherspoon, Westley Weimer, Chris Wells, and Ben Zhao. OceanStore: An Architecture for Global-Scale Persistent Storage. In Proceedings of the 9th international conference on Architectural support for programming languages and operating systems (ASPLOS 2000), pages 190-201, Cambridge, MA, USA, November 2000.

[25] Dahlia Malkhi and Michael Reiter. Byzantine Quorum Systems. The Journal of Distributed Computing, 11(4):203-213, October 1998.

[26] Nikolaos Michalakis, Dah-Ming Chiu, and David S. H. Rosenthal. Long Term Data Resilience Using Opinion Polls. In 22nd IEEE Intl. Performance Computing and Communications Conference, Phoenix, AZ, USA, April 2003.

[27] Dru Mogge. Seven Years of Tracking Electronic Publishing: The ARL Directory of Electronic Journals, Newsletters and Academic Discussion Lists. Library Hi Tech 17, no. 1 (1999): 17-25, 17:17-25, 1999.

[28] David Moore, Geoffrey M. Voelker, and Stefan Savage. Inferring Internet Denial-of-Service Activity. In Proceedings of the 10th USENIX Security Symposium, August 2001.

[29] Michael Reiter. Secure Agreement Protocols: Reliable and Atomic Group Multicast in Rampart. In Proceedings of the 2nd ACM Conference on Computer and Communications Security, pages 68-80, Fairfax, VA, USA, November 1994.

[30] David S. H. Rosenthal and Vicky Reich. Permanent Web Publishing. In Proceedings of the USENIX Annual Technical Conference, Freenix Track (Freenix 2000), pages 129-140, San Diego, CA, USA, June 2000.

[31] A. Rowstron and P. Druschel. Storage Management and Caching in PAST, A Large-scale, Persistent Peer-to-peer Storage Utility. In Proceedings of the Eighteenth Symposium on Operating Systems Principles, October 2001. 
[32] Stuart Staniford, Vern Paxson, and Nicholas Weaver. How to 0wn the Internet in Your Spare Time. In Proceedings of the 11th USENIX Security Symposium, August 2002.

[33] US Govt. Web Design and Usability Guidelines. http://usability.gov/guidelines/softhard.html

[34] Wietse Venema. Murphy's Law and Computer Security. In Proceedings of the 6th USENIX Security Symposium, San Jose, CA, USA, July 1996.

[35] Marc Waldman and David Mazières. Tangler: A Censorship-Resistant Publishing System Based On Document Entanglements. In Proceedings of the 8th ACM Conference on Computer and Communications Security, November 2001.

[36] Matthew Williamson. Throttling Viruses: Restricting Propagation to Defeat Malicious Mobile Code. In Proceedings of the 18th Annual Computer Security Applications Conference, Las Vegas, Nevada, USA, December 2002.

[37] Nick Wingfield. EBay's Figurine Scandal: Auction Site Merchant Disappears With The Goods. Wall Street Journal, Feb 22nd, 2002.

\section{APPENDIX A}

\section{ECONOMIC CONSIDERATIONS}

We use a memory-bound function (MBF) scheme due to Dwork et al. [17]. Here we briefly describe it and compute appropriate costs to impose on poll initiation and voting.

\section{A.1 Overview of a Memory Bound Function Scheme}

The goal of the MBF is to cause the prover of the necessary effort to incur a number $C$ of cache misses and thus RAM accesses. If each of these takes $t$ seconds, the prover must have used $P \cdot t$ seconds on a real computer. Memory bandwidths vary significantly less among commonly available architectures than CPU speeds do, making MBFs superior in provable effort to the CPU-bound functions previously proposed [18], [13].

The scheme we use has two adjustable parameters, the cost of verifying an effort proof $l$ and the ratio between $l$ and the cost of constructing the proof $E$. We measure all costs in cache misses, so a proof costs $E \cdot l$ cache misses to construct and $l$ to verify.

Dwork et al. describe an MBF scheme using an incompressible fixed public data set $T$ larger than any cache it is likely to meet. In our case a gigabyte would be practical. An effort prover who must expend effort $E \cdot l$ is given as challenge a nonce $n$ (so that the prover cannot reuse older effort proofs) and the values of $E$ and $l$. In response, the prover must perform pseudo-random walks of length $l$ in the table $T$, starting from position $s$ of its choosing, computing a one-way value $A$ based on $n, s$ and the encountered elements of table $T$. The walk itself is also dependent on $n$ and $s$. These walks are constructed so that each step will cause an L1 cache miss.

The prover stops when he computes a value $A$ that has 0 bits in its least significant $\log _{2} E$ positions. He is expected to try $E$ walks with different starting positions $s$ before finding an appropriate starting point $s_{0}$, costing $C=E \cdot l$ cache misses.

The $s_{0}$ that yielded the appropriate $A$ is the effort proof. The verifier need only perform the random walk on $T$ starting with the $n$ he chose and the $s_{0}$ sent by the prover, costing $V=l$ cache misses. If the resulting $A$ has the proper 0-bits in its last $\log _{2} E$ positions, the verifier accepts the effort.

We use this scheme to impose costs on voting and poll initiation. Now we describe how we choose appropriate parameters for each phase.

\section{A.2 Choice Of Voting Effort Sizes}

As described in Sections 5.5 and 5.6.1 each round of vote construction and verification processes twice as many document blocks as its predecessor, $2^{i-1}$ blocks in round $i$, or $r=\left\lceil\log _{2}(B+1)\right\rceil$ rounds for a document of $B$ blocks. In each round some $\mathrm{MBF}$ effort is constructed or verified, and part of the document hashed. Let $C_{i}^{M B F}$ and $V_{i}^{M B F}$ be the costs of the $i$-th round MBF construction and verification, respectively. If there are $b$ cache lines in a document block, then the $i$-th round hashing costs $H_{i}=2^{i-1} b$. and is the same in construction and in verification. Then overall voting operations cost $C^{v}=\sum_{i=1}^{r} C_{i}^{v}$ for construction, and $V^{v}=\sum_{i=1}^{r} V_{i}^{v}$ for verification. The cost to verify a disagreeing vote may be less, since hashing stops after the first disagreeing block.

We require (Section 5.1) that the cost of constructing a vote be greater or equal than the cost of verifying that vote, even if the vote is corrupt or malformed, in that in round $i$ either its $\mathrm{MBF}$ verification fails or its document hash disagrees. We examine the four cases in which our requirements must hold: valid agreeing, valid disagreeing, invalid $i$-th MBF proof, and invalid $i$-th document hash. We hold $E$ constant and set $l$ to the size of the document block in each round that is $l_{i}=2^{i-1} b$. Different choices for $l_{i}$ and even variable $E$ for each voting round are possible.

In what follows, we elide the (very simple) algebraic manipulations. The full derivations of Inequalities 5 6 and 7 can be found in the extended version of the paper.

A.2.1 Valid Agreeing Vote: The verifier performs both the $\mathrm{MBF}$ and document hashing components of every verification round. As a result, we must establish that $C^{v} \geq V^{v}$ :

$$
\begin{array}{rlrl}
C^{v} & \geq V^{v} \\
\Leftrightarrow & \sum_{i=1}^{r} C_{i}^{v} & \geq \sum_{i=1}^{r} V_{i}^{v} \\
\Leftrightarrow & \sum_{i=1}^{r} C_{i}^{M B F} & \geq \sum_{i=1}^{r} V_{i}^{M B F} \\
\Leftrightarrow & \sum_{i=1}^{r} E l_{i} & \geq \sum_{i=1}^{r} l_{i} \\
\Leftrightarrow & E & \geq 1
\end{array}
$$

A.2.2 Valid Disagreeing Vote: If we satisfy the inequality 5 for agreeing votes we fulfill the requirements for disagreeing votes, because if the document hash disagrees in round $i$ no further hashing is performed. Thus the cost of verifying in this case is no greater than $V^{v}$.

A.2.3 Invalid $i$-th MBF Proof: If the vote's MBF verification fails in round $i$ its constructor must have performed at least the effort of the preceding rounds $C_{i}^{v, i n v M B F}=\sum_{k=1}^{i-1} C_{k}^{v}$. The verifier's effort ceases after round $i$, costing no more than $V_{i}^{v, i n v M B F}=V_{i}^{M B F}+\sum_{k=1}^{i-1} V_{k}^{v}$. The economic requirements mean that $C_{i}^{v, i n v M B F} \geq V_{i}^{v, \text { invMBF }}$, which is equivalent to

$$
E \geq \frac{1}{2^{i-1}-1}
$$

provided that $2^{i-1}>1$, which holds for all $i>1$. 
For the first voting round $(i=1)$ it is unsatisfiable. Even if the malicious voter sends garbage the verifier must perform some effort, the first round, to eliminate it. Sending garbage costs the verifier $V_{1}^{M B F}=2 b$. But to be invited to send garbage, the malicious peer had to be in the initiator's reference list, which cost at least $C^{v}=(E+1) S$. Sending garbage squanders this effort, $(E+1)$ times the hash cost of the entire document, to impose a cost of two cache misses.

A.2.4 Invalid $i$-th Document Hash: If the $i$-th document hash fails to agree, the vote may still be valid but disagreeing. It is only known to be invalid when the next round MBF verification fails. In this case the vote construction cost at least $C_{i}^{v, \text { invHash }}=C_{i}^{M B F}+\sum_{k=1}^{i-1} C_{k}^{v}$, the vote verification cost $V_{i}^{v, \text { invHash }}=V_{i+1}^{M B F}+\sum_{k=1}^{i} V_{k}^{v}$. We require $C_{i}^{v, \text { invHash }} \geq$ $V_{i}^{v, i n v H a s h}$ :

$$
\begin{array}{cccc}
C_{i}^{v} \text {,invHash } & \geq V_{i}^{v, \text { invHash }} \\
\Leftrightarrow & C_{i}^{M B F}+\sum_{k=1}^{i-1} C_{k}^{v} & \geq V_{i+1}^{M B F}+\sum_{k=1}^{i} V_{k}^{v} \\
\Leftrightarrow & E b 2^{i-1}+\sum_{k=1}^{i-1}\left(C_{k}^{v}-V_{k}^{v}\right) & \geq b 2^{i}+V_{i}^{v} \\
\Leftrightarrow & E b 2^{i-1}+\sum_{k=1}^{i-1}\left(E l_{k}-l_{k}\right) & \geq b 2^{i}+2 b 2^{i-1} \\
\Leftrightarrow & E b 2^{i-1}+(E-1) b \sum_{k=1}^{i-1} 2^{k-1} & \geq 4 b 2^{i-1} \\
\Leftrightarrow & E 2^{i-1}+(E-1)\left(2^{i-1}-1\right) & \geq 4 \cdot 2^{i-1} \\
\Leftrightarrow & E\left(2 \cdot 2^{i-1}-1\right) & \geq 5 \cdot 2^{i-1}-1 \\
\Leftrightarrow & & E & \geq \frac{5 \cdot 2^{i-1}-1}{2 \cdot 2^{i-1}-1}
\end{array}
$$

provided that $2^{i}>1$, which is true for all $i>0$.

A.2.5 Choice for $E$ : Any choice of $E$ that satisfies Inequalities 5 6 and 7 for $i>1$ is appropriate given our economic requirements. Note also that different choices for $l_{i}$ and even variable $E$ for each voting round are also possible. For our choice of $l_{i}, E=4$ is the minimum value for $E$. Higher values might be desirable for smaller document sizes to make rate limitation more effective. If $S$ is the size of the document in cache lines and $E=4$, the cost of constructing a vote is $C^{v}=5 S$, the cost of verifying an agreeing vote $V^{v}=2 S$ and the cost of verifying a disagreeing vote $V^{v d}=S$.

\section{A.3 Designation Of Poll Initiation Effort Sizes}

We want the cost $C^{p}$ of poll initiation per invitee to be at least the induced cost on the invitee:

$$
C^{p} \geq V^{p}+C^{v}
$$

where $V^{p}$ is the cost of verifying the poll initiation effort.

Based on the analysis of the voting costs above, this means

$$
\begin{aligned}
E^{p} l^{p} & \geq l^{p}+5 S \\
\Leftrightarrow \quad\left(E^{p}-1\right) l^{p} & \geq 5 S
\end{aligned}
$$

One choice for the MBF parameters is $E^{p}=4$ and $l^{p}=$ $(5 / 3) S$. The poll initiator must expend $C^{p}=(20 / 3) S$ cache misses per invitee, and each invitee must spend $V^{p}=(5 / 3) S$ cache misses to verify the invitation.

\$Id: 1.145 2003/03/25 18:41:55 maniatis Exp \$ 